\title{
Research on Monitoring and Prewarning System of Accident in the Coal Mine Based on Big Data
}

\author{
Xu Xia $\mathbb{D}^{1},{ }^{1,2}$ Zhigang Chen $\left(\mathbb{D},{ }^{1}\right.$ and Wei Wei $\mathbb{D}^{3}$ \\ ${ }^{1}$ School of Software, Central South University, Changsha, China \\ ${ }^{2}$ Hunan Vocational Institute of Safety Technology, Changsha, China \\ ${ }^{3}$ School of Computer Science and Engineering, Xian University of Technology, Xi'an 710048, China \\ Correspondence should be addressed to Zhigang Chen; czg@csu.edu.cn
}

Received 25 October 2017; Accepted 5 December 2017; Published 6 March 2018

Academic Editor: Wenbing Zhao

Copyright (C) $2018 \mathrm{Xu}$ Xia et al. This is an open access article distributed under the Creative Commons Attribution License, which permits unrestricted use, distribution, and reproduction in any medium, provided the original work is properly cited.

\begin{abstract}
More and more big data come from sensor nodes. There are many sensor nodes placed in the monitoring and prewarning system of the coal mine in China for the purpose of monitoring the state of the environment. It works every day and forms the coal mine big data. Traditional coal mine monitoring and prewarning systems are mainly based on mine communication cable, but they are difficult to place at coal working face tunnels. We use WSN to replace mine communication cable and build the monitoring and prewarning system. The sensor nodes in WSN are energy limited and the sensor data are complicated so it is very difficult to use these data directly to prewarn the accident. To solve these problems, in this paper, a new data aggregation strategy and fuzzy comprehensive assessment model are proposed. Simulations compared the energy consumption, delay time, cooperation cost, and prewarning time with our previous work. The result shows our method is reasonable.
\end{abstract}

\section{Introduction}

Coal is the main energy source in China. According to 2016 National Economic and Social Development Statistical Bulletin, the coal consumption in 2015 is about $64 \%$ of total energy consumption. So the government of China is paying more and more attention to safety production and proposing to use WSN, big data, IOT, and AI technologies to build "digital coal mine" so as to improve the safety level of coal mine industry. In China, most of the coal mining has happened underground, so it is very complicated about the environment in the coal mine. Some gases such as $\mathrm{CH} 4$ and $\mathrm{CO}$ are easy to gather in the coal mine tunnels; it is the main reason causing explosion accident; many workers lose their life. Since 2010, all of the coal mine industries were asked to install the monitoring system to prevent the happening of the accident. The monitoring system is working 24 hours a day without interruption. It means there are lots of monitoring data produced in the monitoring system every day. These data mainly include the state of equipment, the concertation of gas, the pressure of roof, the speed of the wind, and so on.
These data have the characteristics of big data: large data, many types, high velocity, high value, and complex processing process [1]. Taking the data obtained by monitoring system by the State Administration of Work Safety in 2015 as an example, the cumulative information exceeds 5 million and the space occupied is $10 \mathrm{~TB}$ [2]. We can use these data to build the suitable prewarning model and decrease the accident. It is meaningful to society.

The traditional communication method of the monitoring system is burying mine communication cable underground, but it is difficult to do at some places such as coal working face because the coal working face always changes with the digging process. With the development of WSN, its characteristics were proved to easily be used in industry [35]. For these reasons, many scholars proposed using WSN to replace the mine communication cable of the monitoring system. Obviously, the features of coal mine industry are much different with other fields when we use WSN; the coal mine tunnels are very long and narrow. For example, in the mines of the Nanyang Coal Industry Co., Ltd., Hengyang, China, the main haulage roadway is approximately $12,000 \mathrm{~m}$ 


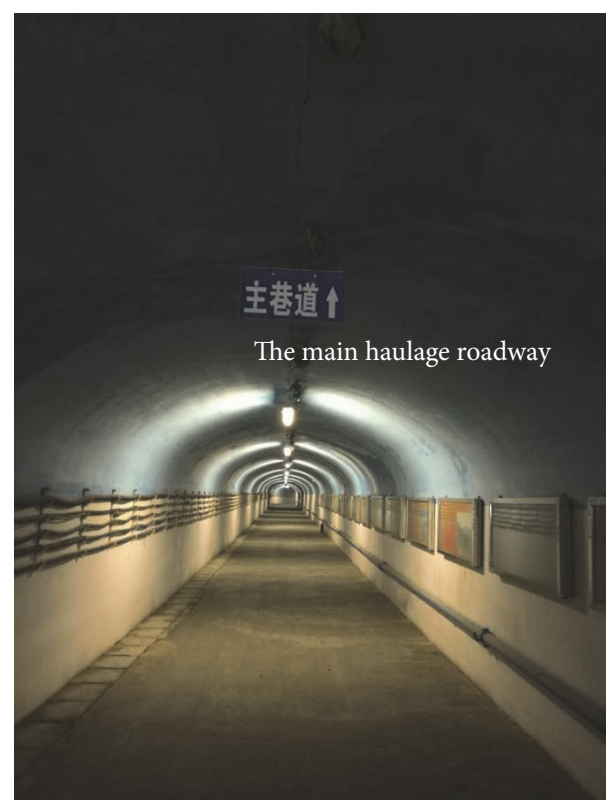

FIGURE 1: The main haulage roadway.

long, and most return airways have lengths of more than $1000 \mathrm{~m}$, but the width is only several meters (Figure 1 shows part of the main haulage roadway). If we want to use WSN in the monitoring system in the coal mine industry, we should solve some problems. The big problem is how to extend the lifetime because it is difficult to change the battery of sensor.

The monitoring system of coal mine almost works every day. So it can produce big data. If we use these data reasonably, we can know the accident in advance and take some measurement to prevent the happening of the accident. Reference [6] proposed if we want to use big data in the coal mine industry with WSN, we should consider how to decrease the energy consumption. Reference [7] proposed that data aggregation can be used in WSN and saved more energy. Based on above research work, this paper will focus on how to use WSN in the monitoring and prewarning system based on big data in coal mine industry.

This paper is organized as follows: Section 2 describes related studies. Section 3 explains the design of the monitoring and prewarning system. Section 4 presents the data aggregation strategy and prewarning model. Section 5 presents the simulation and analyzes the performance. The final section provides the conclusion and future research directions.

\section{Related Studies}

Big data is not a new concept now. But earlier, the big data was limited to some specific organizations like Google, Microsoft, Yahoo, and so on. However, with the developments of IOT, cloud computing and sensor network, the cost of hardware is decreasing and the storage and processing power is increasing. As a result, many sources such as sensors and applications start to generate more and more data. The organizations tend to store these data easily for a long time because the storage and processing ability are great.

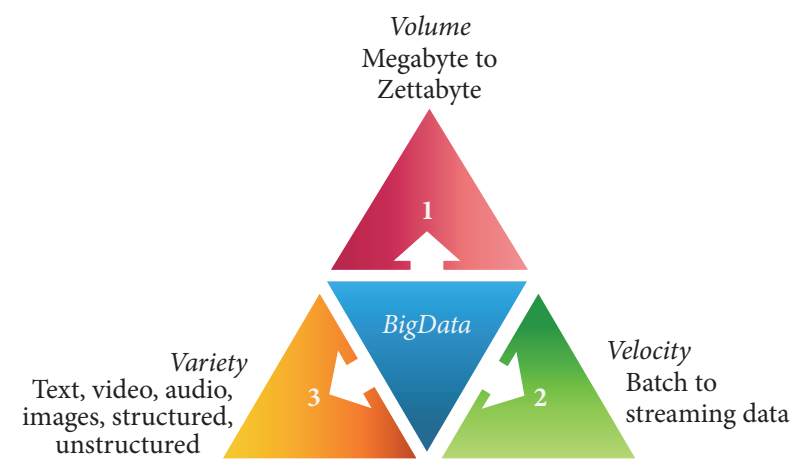

FIGURE 2: Characteristics of big data.

In fact, there is no uniform definition for "big data." The well-known definition is 3V's [8]: volume, variety, and velocity (as shown in Figure 2). EMC [9] has defined big data as any attribute that challenges constraints of a system capability or business need.

More and more big data come from sensor nodes. Reference [10] proposed a greenhouse gas sensor network located throughout California where it collects a large number of real-time data about greenhouse gases and their behavior. The project [11] embedded about 200 sensor nodes on the bridge to monitor the state of the bridge. This monitoring system collects a variety of data including temperature and the pressure of the bridge's concrete reaction to any change. Sensor nodes can collect information in the natural disaster situation in order to optimally utilize the resource and manage supply chains [12]. The challenges in big data mainly include two categories: engineering and semantic [13]. The Jet Propulsion Laboratory (JPL) has identified a number of major challenges in big data [14]; it includes the energy problem especially for the sensor nodes because there are more and more big data coming from sensor nodes in the future.

As to the problem of energy, data aggregation is an efficient method to decrease energy consumption and prolong the lifetime of WSN [15]. To reduce the amount of communication data in WSN, a lot of correlation-based data aggregation methods have been proposed in [7, 16-24]. The traditional data aggregation methods which are used in WSN mainly include two categories, the first type is based on least square method, Bayesian estimation method, D-S evidence theory, and so on; the other is based on artificial intelligence theory of artificial neural network method, fuzzy reasoning method, and rough set method [25]. Reference [26] introduced the data density correlation degree to decrease the amount of data conveyed to sink node, so it can help save more energy.

However, most of the previous studies do not focus on the coal mine. Only a few studies are about coal mining. References [3, 27-30] have studied the energy consumption problem of the coal mine, but all of them did not consider the big data technology and only use single-sink structure. Some novel algorithm is proposed in [31, 32]: in [31] a model for underground mines is generated by adopting a performancebased approach; in [32] a green MAC algorithm is proposed for smart home sensor networks. These strategies 


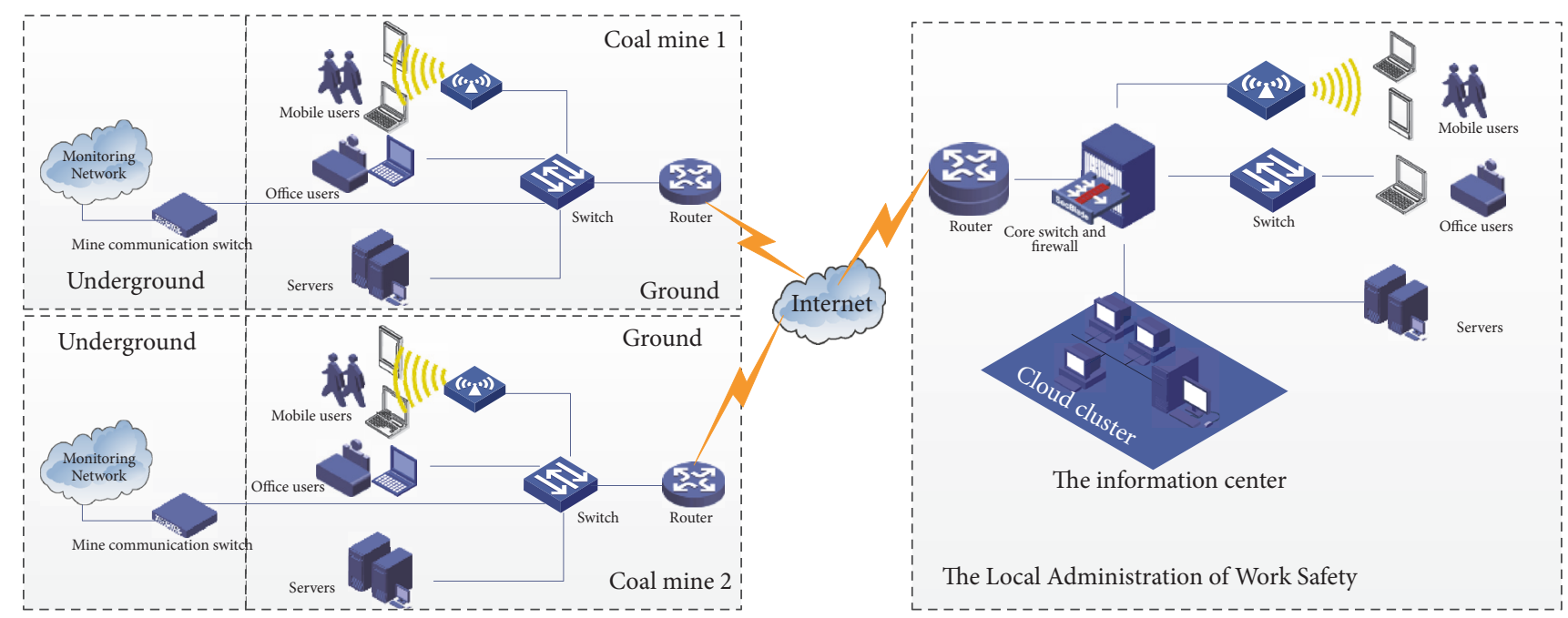

FIgURE 3: The overall structure of system.

effectively extend the network lifetime and improve network performance. Reference [33] introduced IOT and WSN in a mine and described the mine production, monitoring, and prewarning system based on big data technology, but it did not explain how to use sensor nodes and how to build the prewarning model. References [34-44] designed an architecture of monitoring system based on WSN and discussed a data aggregation strategy but did not consider using big data.

Our paper focuses on how to design the monitoring and prewarning system suitable for coal mine industry, how to use data aggregation strategy to decrease energy consumption, and how to build a prewarning model to prevent the accident happening in advance.

\section{Design of the Monitoring and Prewarning System}

3.1. Overall Structure of System. The Local Administration of Work Safety requires knowing the real-time production state of each coal mine, so all of the monitoring data are sent to the information center of the Local Administration of Work Safety. We design the structure of monitoring and prewarning system based on big data which is shown in Figure 3.

The monitoring data are sent to the cloud cluster and form big data; then we can use furry comprehensive assessment model (explained in Section 4.2) to prewarn the problems of the coal mine in advance. The officers and workers in Local Administration of Work Safety and coal mines can gain the prewarning message through their mobile phone or computer, and then they can make some decisions and measures to solve the problems to avoid the accident in advance $[23,30]$.

3.2. Structure of Monitoring Network. The monitoring network is located in underground. There are a large number of monitoring data, mainly the data generated by sensors in the WSN, that are generated in the production process, like the values of voltages, the concentration of gas, the speed of the wind, the pressure of roof, and so on [33]. In our study of the monitoring system, we focus on the monitoring of the concentration of $\mathrm{CH} 4$; it is the main reason for explosion and fire accident in the coal mine. We can use the same method to monitor and process other gases such as $\mathrm{CO}$.

The Coalmining tunnels in China usually are categorized with their functions, including development tunnels, preparation tunnels, and mining tunnels [23, 30, 35-44]. The development tunnels are served for the whole mine, including the horizontal mining area, such as the main haulage roadways and the main return airways. The preparation tunnels are used for digging tunnels such as upward and downward mining areas. The mining tunnels are used to form the coal working face, such as the return airflow roadway and the haulage roadways of coal working face.

The main haulage roadways are relatively wide conveniently to bury mine communication cable. Along the development tunnels, there are many branches; most of them are coal working face tunnels. The coal working face tunnels are narrow, irregular, and always changing with the development of coal mining, so it is difficult to bury mine communication cables; we use WSN to replace the mine communication cable. For these considerations, in our study, the structure of monitoring network is shown in Figure 4.

The WSN is composed of two types of nodes: sink nodes and sensor nodes. The sink nodes are placed near the junction of haulage roadways and coal working face tunnels; it means the number of sink nodes is equal to the number of branches. The sink nodes connect to each other through the mine communication cable. The sensor nodes are placed at the top and the middle of the roof, because the $\mathrm{CH} 4$ is lighter than air and always gather together at the top of the roof. The sensor nodes in each coal working face tunnel have their own ID; the ID number increases along with coal working face tunnel; it means the ID of the first sensor node near the sink node is 1 ; then its neighbor's ID is 2 . In order to gain stable communication performance, we use the Mine Segmenting Wireless Channel Model [31]. The sensor nodes can 


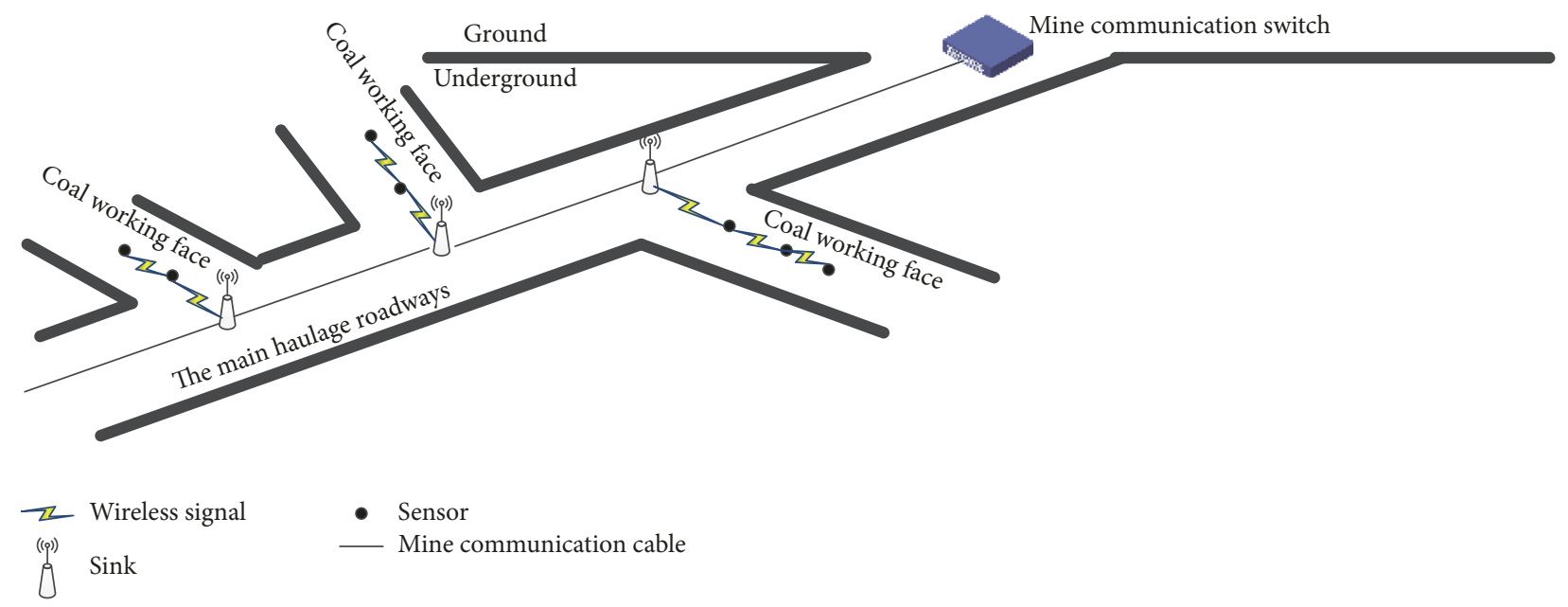

FIGURE 4: The structure of monitoring network.

communicate with neighbor sensor nodes within two hops. The sensor nodes send data to the sink nodes in one or two hops.

In this paper, we suppose that the number of sensor nodes in the network is $N$, and they are distributed in a long-strip region measuring $L \times W$ with $L \gg W$. As soon as the sink nodes and sensor nodes are deployed, the location is fixed and it no longer changes; the output power of sensor nodes is adjustable according to [31]. The sensor nodes are isomorphic with the same initial energy, and they also have data fusion function and self-sensing of residual energy. Furthermore, the energy of sink nodes is unlimited.

3.3. Working Mode. The monitoring network is always in working state for 24 hours without interruption, so the sensor nodes can collect and transfer data permanently; it means the sensor nodes will consume much energy. As we all know that the sensor nodes are powered by the battery which energy is limited and the consumption of energy mainly comes from the communication process. So in our study, we propose the sensor nodes working in two modes; the first mode is decision mode; the second mode is transferring mode. We describe the two modes as follows.

(1) Decision Mode. According to "coal mine safety regulation" which is issued by State Administration of Work Safety in China, there are three important values in the monitoring system, which are alarm value, power-off value, and powerrecovery value; if the concentration is more than the alarm value, the monitoring system will alert to the workers to prevent the concentration from rising and evacuate from their working place; if the concentration is more than the power-off value, all of the electrical equipment will be powered down to prevent the happening of accident; if the concentration is less than the power-recovery value, the monitoring system will give power to the electrical equipment. So it means some data is not important and some data is important. If the sensor nodes only transfer the important data, it will decrease the power consumption largely. So we propose a threshold value
$(E V)$ to help sensor nodes to make a decision; we call this procedure as decision mode. Senor nodes will shut down their communication module, keep collecting environmental parameters, and judge which data should be sent to their neighbor in this mode. When the concentration of $\mathrm{CH} 4$ is larger than $E$, the sensor nodes will be woken up and enter the transferring mode.

(2) Transferring Mode. In this mode, the sensor nodes will send or receive data. Because the structure of monitoring network is shown in Figure 4, the data will only be sent forward and from the sensor node whose ID is larger than another sensor node. Each sensor node can send its data to next sensor node in two hops; to prolong the network life, we propose the cooperation decision mechanism (explained in Section 4.1.2), to help the sensor node decide which node it will send. After that, the sensor node will send the important data to the selected next sensor node until arriving to the sink node.

\section{The Data Aggregation Strategy and Prewarning Model}

In our study, we propose a data aggregation strategy and a fuzzy comprehensive assessment model based on big data in the coal mine industry.

The data aggregation strategy is used in the process of data transformation from one sensor node to the next sensor node and eventually arrives to the sink node in underground. The fuzzy comprehensive assessment model is used in the prewarning system on the ground to prevent the happening of the accident.

4.1. Data Aggregation Strategy. We propose this strategy that mainly takes into consideration the limited power capacity of the sensor nodes and tends to extend the lifetime of the WSN. First of all, the sensor node collects the data and estimates the importance of the data locally; thus it prohibits communications corresponding to unimportant or 


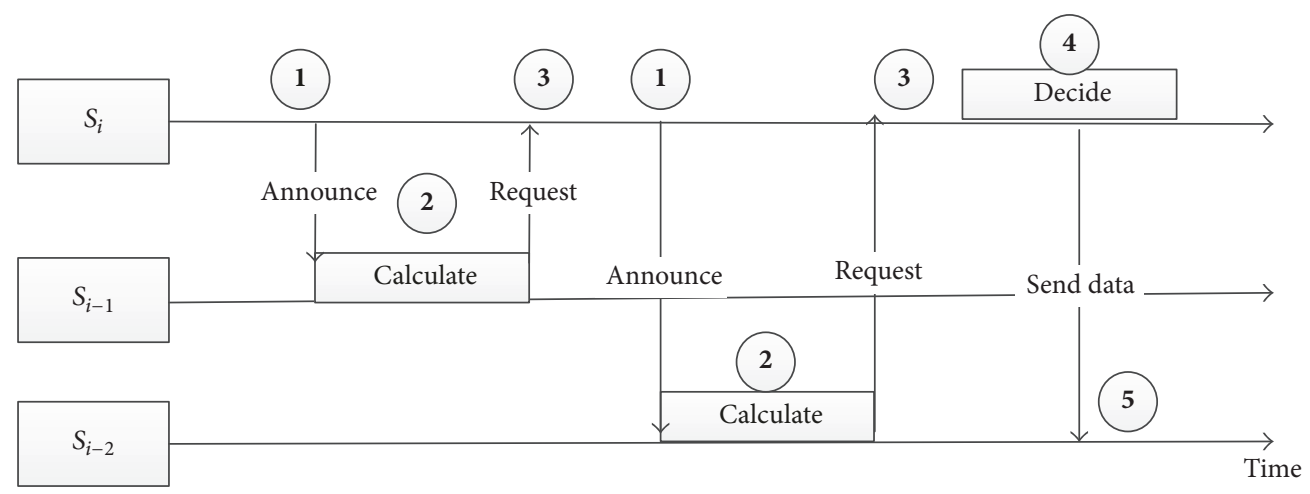

FIgURE 5: Negotiation process among sensor nodes.

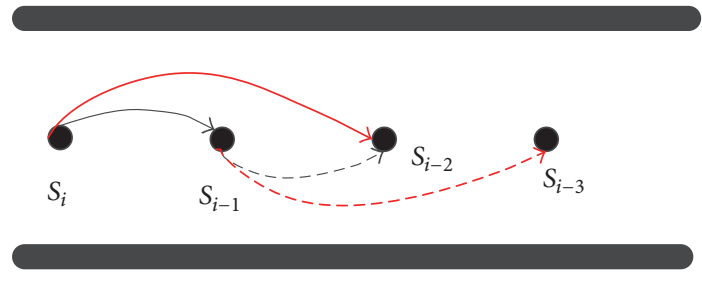

Figure 6: Light load rule.

redundant data. When the sensor node $s_{i}$ (originator node) detects important data, it will send a message to wake up and ask its closest neighbors in two hops $\left(s_{i-1}\right.$ and $\left.s_{i-2}\right)$ to cooperate with it. Neighbors decide to cooperate or not, according to their interests, which are defined by a cooperation decision mechanism (explained in Section 4.1.2). The sensor node $s_{i}$ (originator node) will choose the best neighbor to cooperate and send data to it. Figure 5 shows the overall negotiation process.

4.1.1. Overall Negotiation Process among Sensor Nodes. As Figure 5 shows, the process consists of five steps. $s_{i}$ is the first sensor node which detects important data; it will send an announcement message to $s_{i-1}$ and $s_{i-2}$ (step 1); the two neighbors will calculate their cooperate relevance $(R)$ according to cooperation decision mechanism (explained in Section 4.1.2). After their calculation, they will send $R$ to $s_{i}$ (step 3). $s_{i}$ receives $R$ and selects the larger one as the next cooperation sensor node (step 4 ). We assume $s_{i-2}$ has the larger $R$, so $s_{i}$ will send data to $s_{i-2}$.

In some special circumstances, the sensor node should send important data to its neighbor node and receive the announcement message to cooperate with other nodes at the same time. It is shown in Figure 6 that the sensor nodes $s_{i-1}$ and $s_{i}$ detect important data, so $s_{i-1}$ needs not only to send but also receive the announcement message. In this condition, we will have a rule that the data should always be sent to the light load sensor node. It means $s_{i}$ will send data to $s_{i-2}$ and $s_{i-1}$ will send data to $s_{i-3}$ (along with the bold line).

4.1.2. Cooperation Decision Mechanism. In this section, we explain the mechanism used in the transferring mode to calculate the cooperate relevance $(R)$ by sensor nodes. When the sensor node wants to send important data to its neighbor nodes, it should choose the better one from them, considered to prolong the lifetime of WSN; we define 4 parameters that may have a large influence on the network. These parameters are as follows: the energy $(E)$, the density $(D)$, the position $(P)$, and the data important degree $(I)$. We use (1) [24] to calculate $R$. These parameters will be explained in detail later in this section.

$$
R=E \times \delta_{e}+\frac{1}{D} \times \delta_{d}+P \times \delta_{p}+I \times \delta_{i},
$$

where $\delta_{e}, \delta_{d}, \delta_{p}$, and $\delta_{i}$ are the important factors for the energy, the density, the position, and the data important degree, respectively.

(1) Energy. It is the most important parameter in WSN because the sensor nodes are energy limited. If the sensor node is power exhausted, it will decrease the lifetime of WSN. So we use $E$ to represent the residual energy level. If the sensor node has more residual energy, it is advised to participate in cooperation; another sensor node will save more energy, so it extends the whole lifetime of WSN. We use (2) to calculate $E$; $E_{r}$ refers to the residual energy; $E_{0}$ refers to the initial energy.

$$
E=\frac{E_{r}}{E_{0}}
$$

(2) Density. The density is the number of sensor nodes per square meter. We will consider the number of neighbor sensor nodes within its radio range according to [31]. If the sensor node has more neighbors within its radio range, it means the value of $D$ is bigger, the distance between two sensor nodes is shorter, and the energy consumption will be less, so it is advised to participate in cooperation. That is why, in (1), we take the inverse of the density $(D)$ to calculate. We define the density $(D)$ with

$$
D=\frac{N_{r} /\left(\pi \times r^{2}\right)}{N_{\text {ideal }} /\left(\pi \times r^{2}\right)}=\frac{N_{r}}{N_{\text {ideal }}}
$$

where $r$ refers to the radio range of the sensor node, $N_{r}$ refers to the number of sensor nodes within the radio range, and 


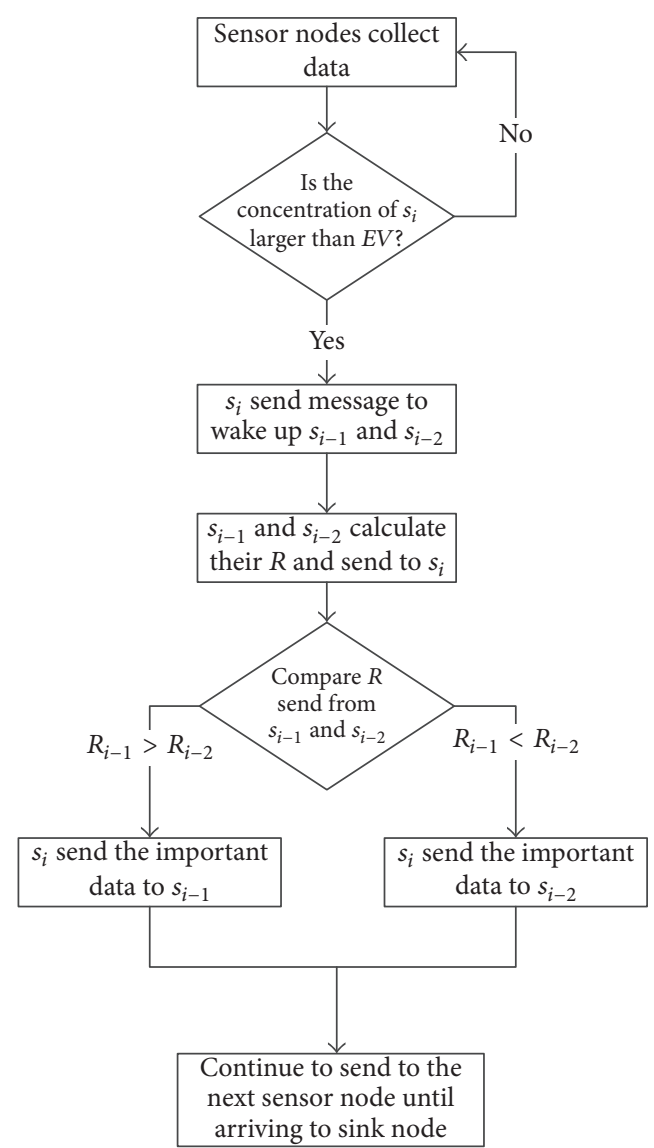

FIGURE 7: The process of data aggregation strategy.

$N_{\text {ideal }}$ refers to theoretical number of sensor nodes and it is given from the ideal distribution of sensor nodes according to [31]. In the ideal case, $N_{r}$ should be equal to $N_{\text {ideal }}$.

(3) Position. There are two types of positions in WSN. The first position is the normal position, where the sensor node has multiple neighbors. The second position is the edge sensor node, which stays at the edge of the network. In fact, only two sensor nodes are belonging to the second position; they are the sensor nodes which have the largest ID and the smallest ID. We define the position as the distance between the sensor node with sink node in the same coal working face.

(4) Data Important Degree. This parameter depends on the running application and the trend of concentration changing of $\mathrm{CH} 4$. It is calculated by local processing, where the sensor node estimates the data important degree according to the rule. For example, if the increment of concentration is growing in 6 hours, it indicates some dangerous thing will happen even the concentration is less than the threshold value $(E V)$. The data will be estimated as important; otherwise, the data is considered unimportant. Figure 7 describes the process of data aggregation strategy.

4.2. Fuzzy Comprehensive Assessment Model. There are many types of sensor nodes in the coal mine. We focus on the concentration of $\mathrm{CH} 4$ in the monitoring system, but there are numerous factors that may give influence to the safety of coal mine, because the environment underground is very complicated. If we use single-layer evaluation model in the prewarning system, the incorrect divide will happen because some key parameters may be neglected. It is similar with the mode proposed by [45], so we use it as a reference and propose our fuzzy comprehensive assessment model.

According to "coal mine safety regulation," the different place has different requirements. Coal working face and return airway are the most dangerous places; most explosions and roof accidents have happened in this place. The main haulage roadways are relatively safe because the air is fresh and the geological conditions are better than other places. Reference [36] sums up and analyzes the main reasons of gas, roof, transportation, floods, and fire accident, so we propose a fuzzy comprehensive assessment model in this section.

The monitoring system includes environmental monitoring, equipment operation status monitoring, coal mine transportation monitoring, and so on. We take the environmental monitoring system as an example; it mainly includes the concentration of $\mathrm{CH}_{4}, \mathrm{CO}, \mathrm{O}_{2}, \mathrm{C}_{2} \mathrm{H}_{2}$, and so on.

Assuming the state factor set of environmental monitoring in the coal mine is $U=\left\{u_{1}, u_{2}, \ldots, u_{n}\right\}, u_{i}(1 \leqslant$ $i \leqslant n)$ is a certain evaluation factor of the environment. The evaluation set of the safety prewarning model is $X=$ $\left\{x_{1}, x_{2}, \ldots, x_{m}\right\} ; x_{i}(1 \leqslant j \leqslant m)$ is the alert level of the coal mine. So the evaluation model is the equal of structuring a mapping rule $f: U \rightarrow X$, making the only sure comments $X_{0}=\left\{x_{1}^{\prime}, x_{2}^{\prime}, \ldots, x_{m}^{\prime}\right\}$ that correspond to the facts $U_{0}=$ $\left\{u_{1}^{\prime}, u_{2}^{\prime}, \ldots u_{n}^{\prime}\right\}$. So, the safety rewarning evaluation model considers various factors and gets the alert level $x_{k}(1 \leqslant k \leqslant$ $m)$.

By using the multilayer comprehensive evaluation model, the state factor set $U$ is divided into $h$ subsets; we call them $U=\left\{u_{1}, u_{2}, \ldots, u_{h}\right\}$ and assume the corresponding evaluation weight matrix is $W=\left\{w_{1}, w_{2}, \ldots, w_{h}\right\}$, in which $W_{i}(i=1,2, \ldots, h)$ is the weight set of each factor subset. Each element is weight set and satisfies the normalization condition $\sum_{i=1}^{h} W_{i}=1 . R(j=1,2, \ldots, h)$ express the fuzzy constraint relationship between each factor subset and evaluation set.

At the beginning, from the first layer, the level 1 assessment $G_{i}=W_{i} \circ R_{i}(i=1,2, \ldots, h)$. Then assemble the $G_{i}$ as the level 2 fuzzy constraint relationship $R$ between factor set and evaluation set. Then step by step evaluate the level set according to the process until arriving to the highest level. The two-layer fuzzy comprehensive evaluation model is shown in Figure 8. The evaluation flow is shown in Figure 9.

\section{Performance Evaluation}

5.1. Simulations. In order to confirm the performance of data aggregation strategy and fuzzy comprehensive assessment model, we use MATLAB and GlomoSim [37, 43, 44] to do some simulations.

In Table 1, we choose the simulation parameters as close as possible to the reality. For example, the sensor nodes 


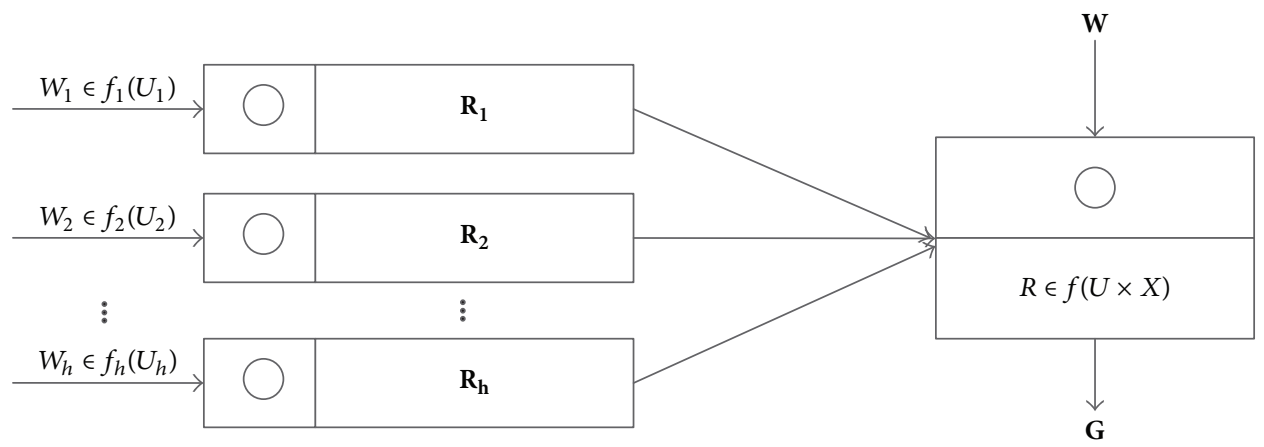

FIgURE 8: Two-layer fuzzy comprehensive evaluation model.

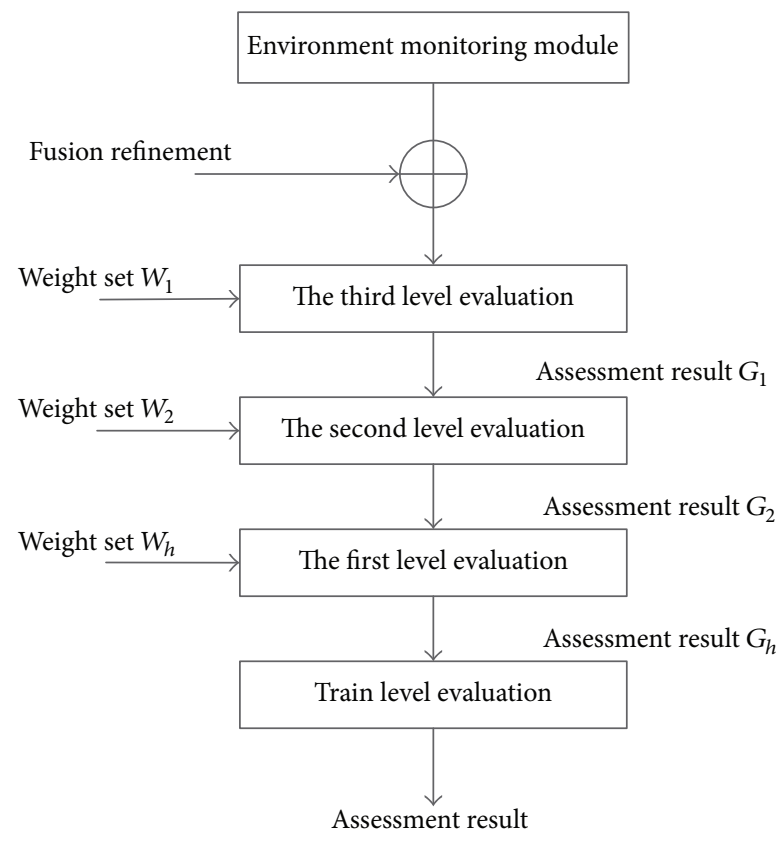

Figure 9: The evaluation flow.

characteristics (transmission, processor, radio range, etc.) are determined according to the specification of $[31,35,39,42]$.

Table 2 resumes the values of importance factor $\delta_{e}, \delta_{p}, \delta_{i}$, and $\delta_{d}$. By giving the same value to $\delta_{e}, \delta_{p}$, and $\delta_{i}$, it means we give the same importance to the energy, the position, and the data importance degree to calculate the cooperate relevance $(R)$. After we make several simulations, we have found that best values for $\delta_{e}$ and $\delta_{d}$ are 0.35 and $\delta_{d}$ is 0.15 . According to the "coal mine safety regulation" and simulations, the threshold value $(E V)$ of $\mathrm{CH} 4$ should be between 0.5 and 1.5 ; for the balance of produce with safety, we choose to fix a threshold of $E V$ to 0.7 .

5.2. Performance Analysis. In this section, we will compare and analyze the energy consumption, the delay, the cooperation cost, and the prewarning time. The goal of data aggregation strategy is to reduce the amount of communicated data, increase the valid data in big data, and hence reduce the energy consumption and prolong the lifetime of the network.
TABLE 1: Simulation parameters.

\begin{tabular}{lc}
\hline Simulation parameters & Values \\
\hline Network coverage & $(0,0)-(1000,20) \mathrm{m}$ \\
Number of sink nodes & $1-6$ \\
Number of sensor nodes & $0-600$ \\
Initial energy of sensor node & $0.5 \mathrm{~J}$ \\
Radio range & $75 \mathrm{~m}$ \\
Throughput & $1 \mathrm{Mbps}$ \\
Simulation time & 48 hours \\
\hline
\end{tabular}

TABLE 2: Cooperate relevance equation parameters.

\begin{tabular}{lc}
\hline Equation parameters & Values \\
\hline$\delta_{e}, \delta_{p}, \delta_{i}$ & 0.35 \\
$\delta_{d}$ & 0.15 \\
\hline
\end{tabular}

The aim of fuzzy comprehensive assessment model is to decrease the time of prewarn.

In the simulation, we compare the data aggregation strategy with PCEB-MS protocol which is proposed in our previous work [35] and give a simulation to prove the fuzzy comprehensive assessment model can reduce the prewarning time compared with the other methods [33].

(1) Energy Consumption. We define the energy consumption as the average value of the energy consumed by each sensor node during their transmit, receive, and process data. We use the classical energy consumption model [39-41] for simulation. Figure 10 shows the comparison between PCEB-MS and our strategy. The result proves that our strategy is significantly better than our previous work. At the beginning, the average consumption of two methods is similar, but with the increase of sensor nodes, our strategy can save more energy; when the number of sensor nodes is 600 , the average energy consumption of PCEB-MS is $15 \times 10^{4}$, but our strategy is only about $10 \times 10^{4}$, so the average energy consumption is reduced by about $34 \%$.

(2) Delay. We define the delay as the average latency needed to send a message from a sensor node which detects important data to the sink; it is including the communication and processing time. At this simulation, we placed 6 sink nodes. 


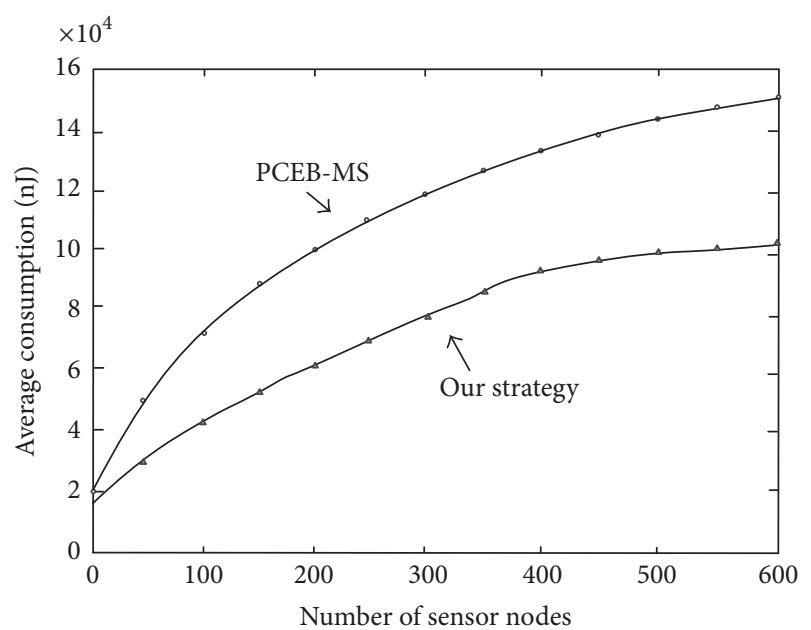

FIGURE 10: Comparison of average energy consumption.

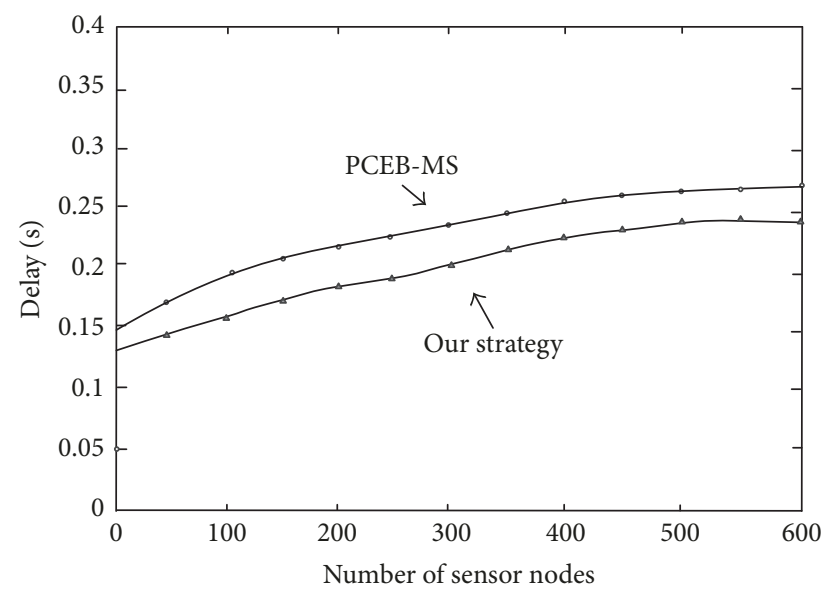

FIGURE 11: Comparison of delay.

Figure 11 shows our strategy needs less time from each sensor node to sink node because relatively the sensor node does not need process complicated data.

(3) Cooperation Cost. When the sensor node needs to send important data, it will wake its neighbor up in two hops and make a decision to choose a neighbor to send data, so it will cause cooperation cost. Figure 12 shows with the increase of the sensor nodes the cooperation cost will decrease and the consumption is very little. It means the cost is valuable.

(4) Prewarning Time. We use the prewarning time to make sure the fuzzy comprehensive assessment model is reasonable. We define it as how long the time is before the accident is prewarned. There are some studies about the prewarning system in China, such as [33, 46, 47], but only [33] has the similar prewarning system structure with our study, so we choose [33] as a comparison. Reference [33] has proposed a data analysis platform to forecast the accident through the IOT and big data technology, but it has not used any prewarning model.
TABLE 3: Comparison result.

\begin{tabular}{lcc}
\hline $\begin{array}{l}\text { Concentration } \\
\text { accident }\end{array}$ & \multicolumn{2}{c}{ Prewarning time } \\
\hline $\mathrm{CH} 4$ & Using our model & Not using our model \\
$\mathrm{CO}$ & 2.5 hours & 2.2 hours \\
$\mathrm{C}_{2} \mathrm{H}^{2}$ & 2.1 hours & 1.5 hours \\
\hline
\end{tabular}

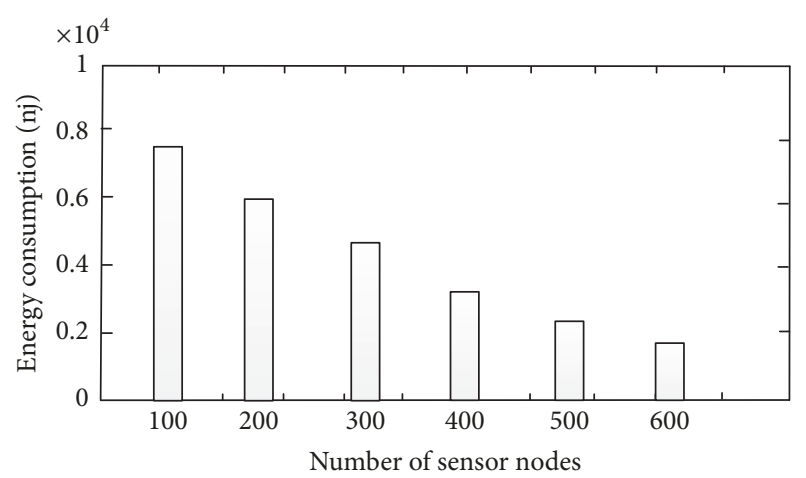

Figure 12: Cooperation cost.

In this simulation, we use the fuzzy comprehensive assessment model on the platform. We get the data from the simulated coal mine, which is a project to train the staff for coal mine industry in our college. Table 3 shows the difference between using this model and not using this model to prewarn of the accident. Obviously, the model can increase about $0.5-1.0$ hour of prewarning time.

\section{Conclusions}

In this paper, we focus on the safety problems of coal mine industry in China. We proposed a monitoring and prewarning system based on big data to prevent happening of the accident. We used the WSN network to replace mine communication cable underground at coal working face and designed the data aggregation strategy and fuzzy comprehensive assessment model to help the system prewarn the accident in advance. At last, we use MATLAB and GlomoSim to do some simulations to make sure our strategy and model are reasonable. Results of simulations show that our method can improve the performance of the monitoring network and prewarning system largely.

\section{Conflicts of Interest}

The authors declare that there are no conflicts of interest regarding the publication of this paper.

\section{Acknowledgments}

This work was supported in part by the National Natural Science Foundation of China (61672540, 61379057), 2015 Prevention of Major Accidents Safety Key Technology Projects (Hunan-0012-2015AQ), and 2017 Hunan Natural Science 
Foundation of China (2017JJ5004). This job is supported by the Open Program of Xiamen Key Laboratory of Computer Vision and Pattern Recognition, Huaqiao University (no. 600005-Z17X0001) and the Specialized Research Fund for the Doctoral Program of Higher Education of China (Grant no. 20136118120010).

\section{References}

[1] W. Haijun and W. Xianli, "Analysis on application of coal mine big data in age of Internet +," Coal Science \& Technology Magazine, vol. 44, no. 2, pp. 139-143, 2016.

[2] "Big Data and Safety Production Review," http://aqscjdglj.tjftz .gov.cn/system//02/10/010073245.shtml.

[3] X. Xia, Z. Chen, D. Li, and W. Li, "Proposal for efficient routing protocol for wireless sensor network in coal mine goaf," Wireless Personal Communications, vol. 77, no. 3, pp. 1699-1711, 2014.

[4] D. Zhang, Z. Chen, M. K. Awad, N. Zhang, H. Zhou, and X. S. Shen, "Utility-optimal resource management and allocation algorithm for energy harvesting cognitive radio sensor networks," IEEE Journal on Selected Areas in Communications, vol. 34, no. 12, pp. 3552-3565, 2016.

[5] D. Zhang, Z. Chen, H. Zhou, L. Chen, and X. Shen, "Energybalanced cooperative transmission based on relay selection and power control in energy harvesting wireless sensor network," Computer Networks, vol. 104, pp. 189-197, 2016.

[6] E. Sun, X. Zhang, and Z. Li, "The internet of things (IOT) and cloud computing (CC) based tailings dam monitoring and prealarm system in mines," Safety Science, vol. 50, no. 4, pp. 811-815, 2012.

[7] C. M. Chao and T. Y. Hsiao, "Design of structure-free and energy-balanced data aggregation in wireless sensor networks," Journal of Network \& Computer Applications, vol. 37, no. 1, pp. 229-239, 2014.

[8] W. Zhao, P. M. Melliar-Smith, and L. E. Moser, "Fault tolerance middleware for cloud computing," in Proceedings of the 3 rd IEEE International Conference on Cloud Computing (CLOUD '10), pp. 67-74, Miami, FL, USA, July 2010.

[9] R. Lun and W. Zhao, "A survey of applications and human motion recognition with microsoft kinect," International Journal of Pattern Recognition and Artificial Intelligence, vol. 29, no. 5, Article ID 1555008, 2015.

[10] “The industry leader in emerging technology research," https:// gigaom.com/archives/energy-environment/.

[11] K. Taylor-Sakyi, "Big Data: Understanding Big Data, 2016.

[12] E. Kinoshita and T. Mizuno, What Is Big Data Big Data Management, Springer International Publishing, 2017.

[13] C. Bizer, P. Boncz, M. L. Brodie, and O. Erling, "The meaningful use of big data: four perspectives-four challenges," ACM SIGMOD Record, vol. 40, no. 4, pp. 56-60, 2011.

[14] D. L. Jones, K. Wagstaff, D. R. Thompson et al., "Big data challenges for large radio arrays," in Proceedings of the 2012 IEEE Aerospace Conference, pp. 1-6, Big Sky, MT, USA, March 2012.

[15] F. Yuan, Y. Zhan, and Y. Wang, "Data density correlation degree clustering method for data aggregation in WSN," IEEE Sensors Journal, vol. 14, no. 4, pp. 1089-1098, 2014.

[16] S. Madden, M. J. Franklin, J. M. Hellerstein, and W. Hong, "TAG: a tiny aggregation service for ad-hoc sensor networks," ACM SIGOPS Operating Systems Review, vol. 36, no. 1, pp. 131$146,2002$.
[17] J. Zheng, P. Wang, and C. Li, "Distributed data aggregation using slepianwolf coding in cluster-based wireless sensor networks," IEEE Transactions on Vehicular Technology, vol. 59, no. 5, pp. 2564-2574, 2010.

[18] M. C. Vuran, Ö. B. Akan, and I. F. Akyildiz, "Spatio-temporal correlation: theory and applications for wireless sensor networks," Computer Networks, vol. 45, no. 3, pp. 245-259, 2004.

[19] J. Yuan and H. Chen, "The optimized clustering technique based on spatial-correlation in wireless sensor networks," in Proceedings of the 2009 IEEE Youth Conference on Information, Computing and Telecommunication (YC-ICT '09), pp. 411-414, Beijing, China, September 2009.

[20] A. Rajeswari and P. T. Kalaivaani, "Energy efficient routing protocol for wireless sensor networks using spatial correlation based medium access control protocol compared with IEEE 802.11," in Proceedings of the 2011 International Conference on Process Automation, Control and Computing (PACC '11), pp. 1-6, Coimbatore, India, July 2011.

[21] J. N. Al-Karaki, R. Ul-Mustafa, and A. E. Kamal, "Data aggregation and routing in wireless sensor networks: optimal and heuristic algorithms," Computer Networks, vol. 53, no. 7, pp. 945-960, 2009.

[22] C. Hua and T.-S. P. Yum, "Optimal routing and data aggregation for maximizing lifetime of wireless sensor networks," IEEE/ ACM Transactions on Networking, vol. 16, no. 4, pp. 892-903, 2008.

[23] Z. Sun, H. Song, H. Wang, and X. Fan, "Energy balance-based steerable arguments coverage method in WSNs," IEEE Access, p. 99, 2017.

[24] A. Sardouk, M. Mansouri, L. Merghem-Boulahia, D. Gaïti, and R. Rahim-Amoud, "Multi-agent system based wireless sensor network for crisis management," in Proceedings of the 53rd IEEE Global Communications Conference (GLOBECOM '10), pp. 1-6, University of Kansas, January 2011.

[25] M. Zhang and M. Shen, "Research of WSN-based data fusion in water quality monitoring," Computer Engineering \& Applications, vol. 50, no. 23, pp. 234-238, 2014.

[26] W. Wei, Q. Xu, L. Wang et al., "GI/Geom/1 queue based on communication model for mesh networks," International Journal of Communication Systems, vol. 27, no. 11, pp. 3013-3029, 2014.

[27] F. Wang, X. Zhang, M. Wang, and G. Chen, "Energy-efficient routing algorithm for WSNs in underground mining," Journal of Networks, vol. 7, no. 11, pp. 1824-1829, 2012.

[28] W. Chen, X. Jiang, X. Li, J. Gao, X. Xu, and S. Ding, "Wireless Sensor Network nodes correlation method in coal mine tunnel based on Bayesian decision," Measurement, vol. 46, no. 8, pp. 2335-2340, 2013.

[29] G. Zhou, L. Huang, and Z. Zhu, "A Zoning Strategy for Uniform Deployed Chain-Type Wireless Sensor Network in Underground Coal Mine Tunnel," in Proceedings of the 2013 IEEE 10th International Conference on High Performance Computing and Communications 2013 IEEE International Conference on Embedded and Ubiquitous Computing (HPCC EUC), pp. 11351138, Zhangjiajie, China, November 2013.

[30] W. Wei, X.-L. Yang, P.-Y. Shen, and B. Zhou, "Holes detection in anisotropic sensornets: topological methods," International Journal of Distributed Sensor Networks, vol. 2012, Article ID 135054, 9 pages, 2012.

[31] W. Farjow, K. Raahemifar, and X. Fernando, "Novel wireless channels characterization model for underground mines," 
Applied Mathematical Modelling, vol. 39, no. 19, pp. 5997-6007, 2015.

[32] S. Latif and X. Fernando, "A greener MAC layer protocol for smart home wireless sensor networks," in Proceedings of the 2013 IEEE Online Conference on Green Communications (OnlineGreenComm '13), pp. 169-174, Piscataway, NJ, USA, October 2013.

[33] C.-M. Li, R. Nie, and X.-Y. Qian, "Forecast and prewarning of coal mining safety risks based on the internet of things technology and the big data technology," Electronic Journal of Geotechnical Engineering, vol. 20, no. 20, pp. 11579-11586, 2015.

[34] Y. Zhang, W. Yang, D. Han, and Y.-I. Kim, "An integrated environment monitoring system for underground coal minesWireless Sensor Network subsystem with multi-parameter monitoring," Sensors, vol. 14, no. 7, pp. 13149-13170, 2014.

[35] X. Xia, Z. Chen, H. Liu, H. Wang, and F. Zeng, "A routing protocol for multisink wireless sensor networks in underground coalmine tunnels," Sensors, vol. 16, no. 12, pp. 2032-2054, 2016.

[36] S. Jiping, "Accident analysis and big data and Internet of Things in coal mine," Industry and Mine Automation, vol. 41, no. 3, pp. $1-5,2015$.

[37] V. Mishra and S. Jangale, "Analysis and comparison of different network simulators," International Journal of Application or Innovation in Engineering \& Management, 2014.

[38] Y. Liu, A. Liu, S. Guo, Z. Li, Y. Choi, and H. Sekiya, "Contextaware collect data with energy efficient in Cyber-physical cloud systems," Future Generation Computer Systems, 2017.

[39] X. Liu, G. Li, S. Zhang, and A. Liu, "Big program code dissemination scheme for emergency software-define wireless sensor networks," Peer-to-Peer Networking and Applications, pp. $1-22,2017$.

[40] X. Chen, M. Ming, and L. Anfeng, "Dynamic Power Management and Adaptive Packet Size Selection for IoT in eHealthcare," Computers \& Electrical Engineering, 2017.

[41] X. Fan, H. Song, and X. Fan, "Imperfect information dynamic stackelberg game based resource allocation using hidden markov for cloud computing," IEEE Transactions on Services Computing, no. 99, p. 1, 2016.

[42] N. Zhang, H. Chen, X. Chen, and J. Chen, "ELM meets urban computing: ensemble urban data for smart city application," in Proceedings of the ELM-2015, vol. 1, pp. 51-63, 2016.

[43] W. Zhao, "A Byzantine Fault Tolerant Distributed Commit Protocol," in Proceedings of the Third IEEE International Symposium on Dependable, Autonomic and Secure Computing (DASC '07), pp. 37-46, Columbia, MD, USA, September 2007.

[44] H. Song, W. Li, and P. Shen, "Gradient-driven parking navigation using a continuous information potential field based on wireless sensor network," Information Sciences, vol. 408, pp. 100-114, 2017.

[45] S. Hou, F. Dou, Y. Li, and Z. Long, "Assessment model of the maglev train braking system safety pre-warning and the optimization of Parameters," in Proceedings of the 28th Chinese Control and Decision Conference (CCDC '16), pp. 4915-4920, May 2016.

[46] L. I. Hao-Min, L. U. Jian-Jun, and C. Wei, "Research of coal mine safety monitoring and early warning system based on cloud computing," Industry and Mine Automation, vol. 39, no. 3, pp. 46-50, 2013.

[47] C. Qinggui, Z. Jing, S. Qihua, and Y. Kai, "Design and Application of Hidden Danger Management and Early-warning System for Coal Mine Accidents," Mining Safety \& Environmental Protection, vol. 43, no. 3, pp. 107-114, 2016. 


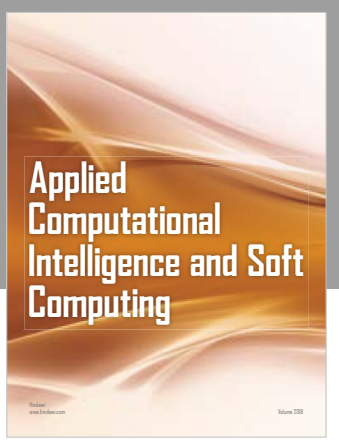

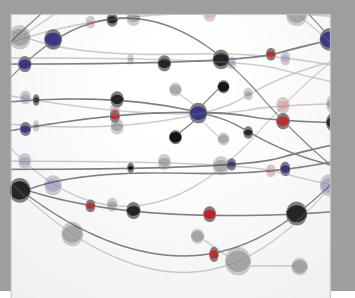

The Scientific World Journal
Submit your manuscripts at

Computing
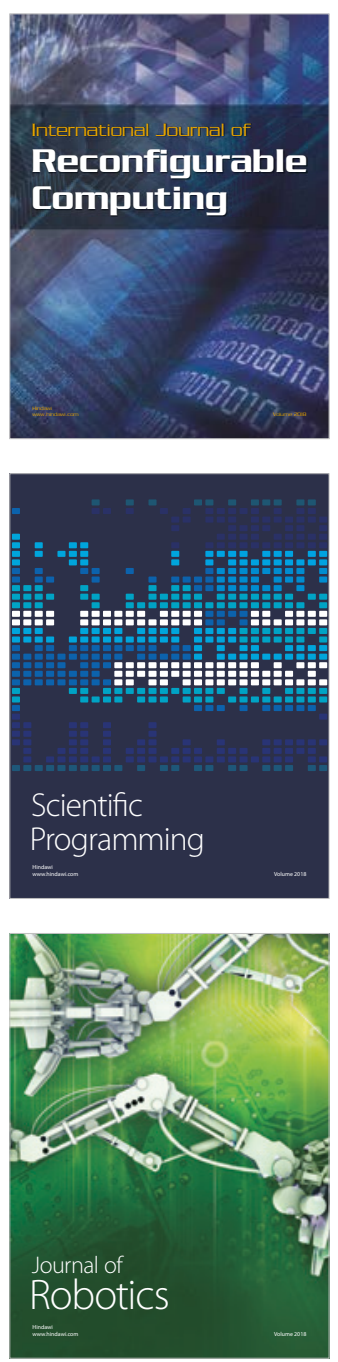

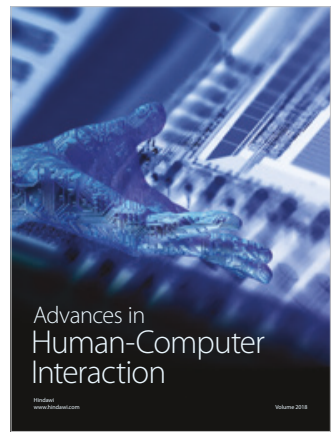

Human-Compute

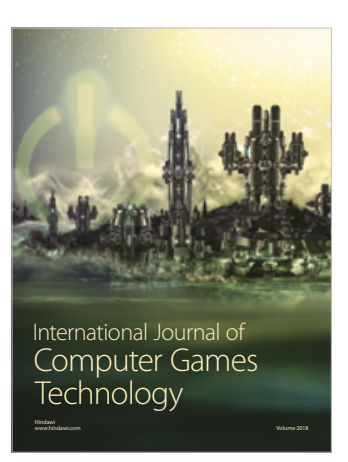

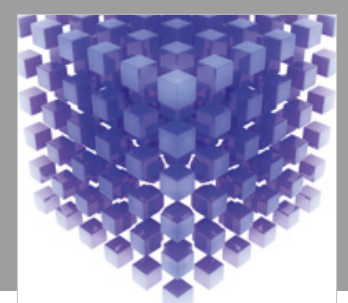

Mathematical Problems in Engineering

\section{Engincering}
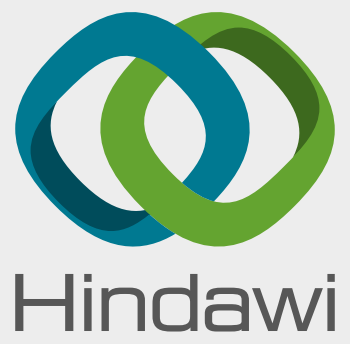

www.hindawi.com
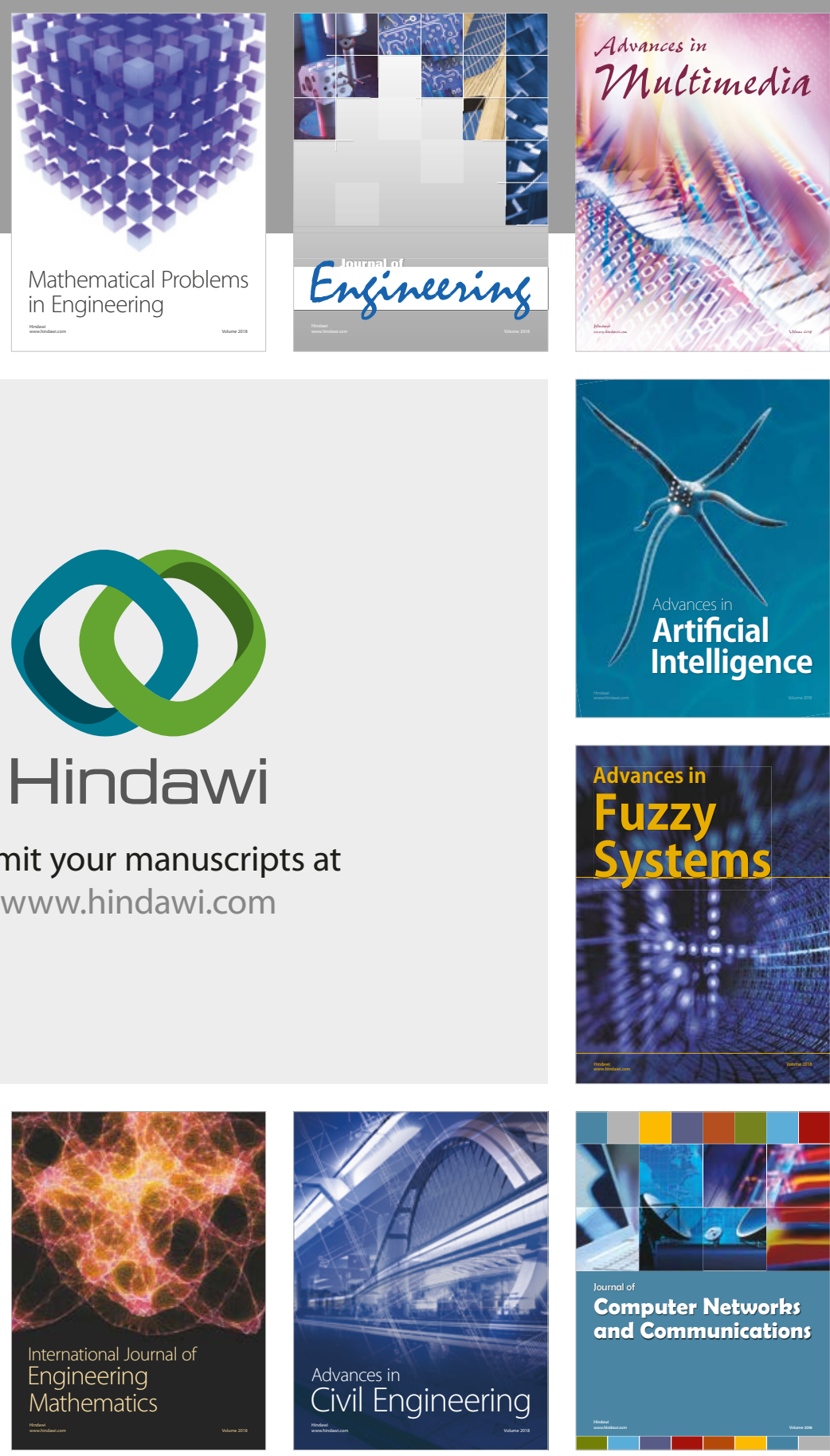

Computer Networks and Communications

Multimedia
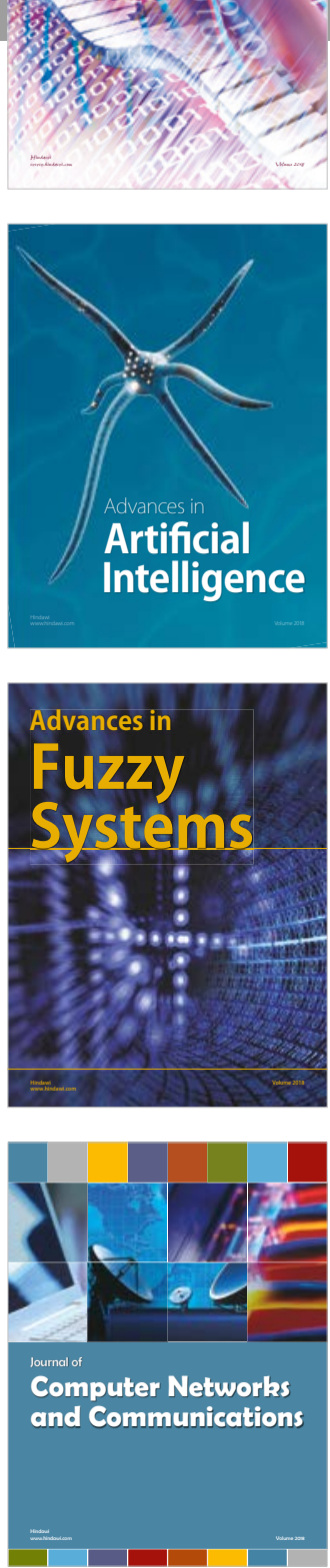

Advances in

Modelling \&

Simulation

in Engineering

interaction

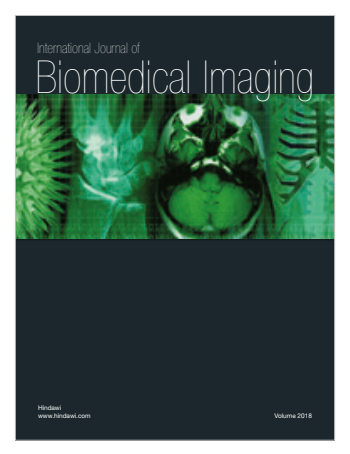

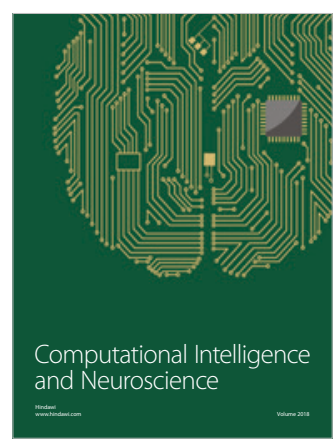

